\title{
IC-Integrated Flexible Shear-Stress Sensor Skin
}

\author{
Yong Xu, Member, IEEE, Yu-Chong Tai, Member, IEEE, Adam Huang, and Chih-Ming Ho, Member, ASME
}

\begin{abstract}
This paper reports the successful development of the first IC-integrated flexible MEMS shear-stress sensor skin. The sensor skin is $1 \mathrm{~cm}$ wide, $2 \mathrm{~cm}$ long, and $70 \mu \mathrm{m}$ thick. It contains 16 shear-stress sensors, which are arranged in a 1-D array, with on-skin sensor bias, signal-conditioning, and multiplexing circuitry. We further demonstrated the application of the sensor skin by packaging it on a semicylindrical aluminum block and testing it in a subsonic wind tunnel. In our experiment, the sensor skin has successfully identified both the leading-edge flow separation and stagnation points with the on-skin circuitry. The integration of IC with MEMS sensor skin has significantly simplified implementation procedures and improved system reliability.

[905]
\end{abstract}

Index Terms-Flexible skin, MEMS-IC integration, shear-stress sensors.

\section{INTRODUCTION}

W ITH the emergence of MEMS technology, numerous miniaturized sensors and actuators have been fabricated using techniques originally developed for the integrated circuits industry. Most MEMS devices are inherently fabricated on rigid substrates. However, for a wide variety of applications, it has long been desirable for sensors, actuators, and circuits to be mounted on nonplanar surfaces or even on flexible objects such as a human body. For example, we are working on a new way of controlling an Unmanned Aerial Vehicle (UAV) through the sensing/controlling of the flow separation on the curved leading edge of the wing. This requires distributed shear-stress sensors mounted on the curved surface of the leading edge to measure the shear-stress profile. We have already developed flexible shear-stress sensor skins for the above mentioned UAV project [1] based on the idea first reported by Barth et al. in 1985 [2]. These skins, however, contained only sensors and required many electrical leads connections. The complete separation-detecting system consisted of the packaged sensor skin, bias board, data acquisition board, and a tremendous number of interconnection cables as shown in Fig. 1. Such a complicated system was not easy to employ and the overall reliability was an issue. It is highly desirable to develop IC-integrated shear-stress sensor skins with on-skin bias circuits, amplifiers, and multiplexers. By making IC-integrated sensor skin, we can eliminate the bias board and interconnection cables, simplify

Manuscript received July 17, 2002; revised April 7, 2003. This work was supported by DARPA under the Navy Contract N66001-97-C-8610 and by the NSF Center for Neuromorphic System Engineering at Caltech. This paper is based upon a 2002 Hilton Head presentation. Subject Editor K. D. Wise.

Y. Xu is with the Department of Electrical and Computer Engineering, Wayne State University Detroit, MI 48202 USA (e-mail: yxu@ece.eng.wayne.edu).

Y.-C. Tai is with the California Institute of Technology, Pasadena, CA 91125 USA.

A. Huang and C.-M. Ho are with the Mechanical and Aerospace Engineering (MAE), University of California, Los Angeles, CA 90024 USA.

Digital Object Identifier 10.1109/JMEMS.2003.815831 the implementation procedures, and improve system reliability as well.

MEMS-IC integration can be generally categorized into two groups: hybrid integration and monolithic integration. In the case of hybrid integration, MEMS parts and circuit parts are fabricated separately and then are packaged together by flip-chip bonding or other packaging technologies. With regard to flexible shear-stress sensor skin, monolithic integration is preferred since hybrid integration does not simplify the packaging process. Based on when MEMS parts are fabricated, monolithic integration can be classified as mixed MEMS-IC process, MEMS-first process (pre-IC or pre-CMOS) and IC-first process (post-IC or post-CMOS). One well-know example of the first approach is Analog Devices' accelerometer, which is fabricated by interleaving and customizing the MEMS manufacturing steps with the IC process [3]. This approach needs to modify the IC fabrication process and the accessibility is limited. Smith et al. at Sandia National Laboratories developed a unique MEMS-first process [4]. In this approach, MEMS devices are first fabricated in a trench on the surface of the wafer. The wafer is then planarized and the trench is sealed. The MEMS processed wafer then undergoes conventional IC process. The accessibility of this approach is very limited as well because most IC foundries do not accept pre-processed wafers. Most monolithic integrations are done with the post-CMOS approach, which takes advantage of the widely available CMOS foundry services. For the present IC-integrated shear-stress sensor skin, post-CMOS process is chosen for the same reason.

The aluminum metallization of ICs limits the temperature of post-IC process to less than $450^{\circ} \mathrm{C}$. This requirement precludes a lot of high temperature deposition processes such as LPCVD nitride and polysilicon in post-IC process. Therefore, we have to utilize the existing materials of IC process, or use some low temperature deposition processes such as sputtering, spin coating, and electroplating. In addition, the integration of MEMS devices and ICs into flexible skins presents a unique challenge. The design, layout, and fabrication process have to be carefully considered to insure good compatibility.

\section{DESIGN}

Local wall shear stress can be measured by many methods, including Stanton tube, direct measurement, thermal method, Preston tube, sublayer fence and electrochemical technique [5]. Of these approaches, the thermal method is most widely used since it does not interfere with the flow and offers the possibility of measuring time-varying flows. Conventional thermal shear-stress sensors are typically made by depositing thin-film metal resistors, usually platinum or nickel, on flat 
Packaged sensor skin

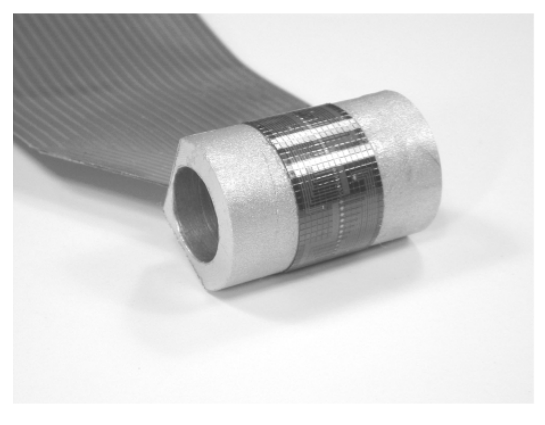

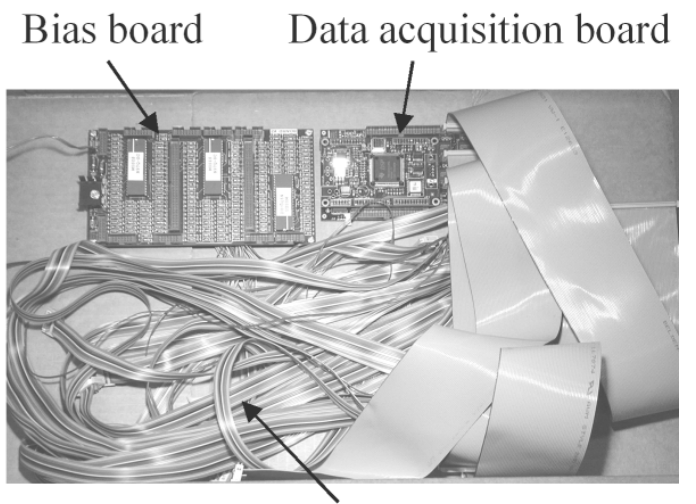

Cables

Fig. 1. Flow separation detection system based on nonintegrated sensor skin.

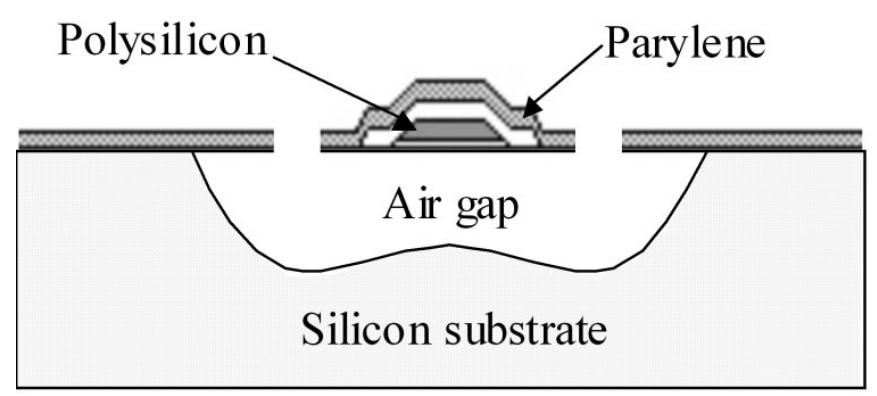

Fig. 2. Cross section of the thermal shear-stress sensor.

substrates. The resistor is electrically heated during operation. The input power of the resistor will change with the wall shear stress from the ambient flow field and this change is readily detected electronically. A large portion of the power is lost to the substrate via thermal conduction. With micromachining technology, a vacuum cavity can be fabricated underneath the resistor to reduce the heat loss to the substrate, thus improving the sensitivity and frequency response of sensor [6]. This is the sensor design used in the nonintegrated sensor skin [1].

The previously used vacuum cavity [6] is difficult to realize by post-CMOS process because it involves many high temperature processes. To circumvent this problem, a different design is employed here [7]. In this design, the gate poly of the CMOS process is utilized as the sensing elements of the shearstress sensors, and parylene is deposited as the membrane material to support the sensing elements. The silicon underneath the sensing element is etched away by $\mathrm{BrF}_{3}$, which is a gas phase silicon etchant [8], to achieve good thermal isolation. The cross section of the sensor is illustrated in Fig. 2. Note that parylene is the generic name for members of a unique family of thermoplastic polymers that are deposited by using the dimer of para-xylylene (di-para-xylylene, or DPXN) [9]. There are three types of parylene commonly used: parylene N, C, and D. The most important advantage of using parylene as a post-IC material is that the parylene deposition is a room temperature conformal process. In addition, parylene has good mechanical prop- erties, can be patterned by oxygen plasma, and is chemically inert.

Fig. 3 shows the schematic of the integrated flexible shearstress sensor skin. The shear-stress sensors are made using the gate polysilicon of the CMOS process as the sensing elements. The resistance of the shear-stress sensor is chosen as $1.2 \mathrm{k} \Omega$. These sensors operate in constant current (CC) mode and are biased by the cascode current mirrors. The sensors are raised to elevated temperatures during operation by Joule heat. The shear stress can be monitored by measuring the voltage change across the sensing element. One important parameter of the operation is resistive over-heat ratio, which is defined as $\left(R-R_{0}\right) / R_{0}$, where $R$ and $R_{0}$ are the resistance of sensing element at operating temperature and reference temperature, respectively. The output voltage is multiplexed by PMOS switches and addressing circuitry. There are 16 shear-stress sensors, which are accessed by a 4-bit address. An operational amplifier is also implemented to perform on-chip amplification.

The layout should be carefully designed, not only for patterns in horizontal dimension, but also for layers in vertical dimension. As shown in Fig. 4, the dimension of the skin is $20 \times 10 \mathrm{~mm}^{2}$. The 2 -D array of small gray squares on the right side represent the $400 \times 400 \mu \mathrm{m}^{2}$ silicon islands with a pitch of $500 \mu \mathrm{m}$. The 16 shear-stress sensors, which are inside the dashed rectangle, are arranged in a 1-D array and placed on every two islands in the central row. The distance between the adjacent two sensors is $1 \mathrm{~mm}$, and the total distance covered by the 16 sensors is $15 \mathrm{~mm}$. These 16 shear-stress sensors are connected to the current mirrors by the second metal layer of the CMOS process. The shared ground wire is placed below the 1-D sensor array. All the electronic parts are placed on the left side of the skin on a big silicon island, which is denoted by the rectangle on the left side of the sensor array. The multiplexer and operational amplifier are placed in the lower-left corner, whereas the cascode current mirrors are placed in the upper-left corner. All the pads are on the left-side edge. If necessary, the electronic parts can also be distributed on the small silicon islands. Between the big silicon island on the left side and the small silicon islands on the right side, there is an area where silicon underneath is completely etched away such that these two parts are mechanically connected by parylene film only. This allows very sharp folding angle when packaging the flexible skin, 


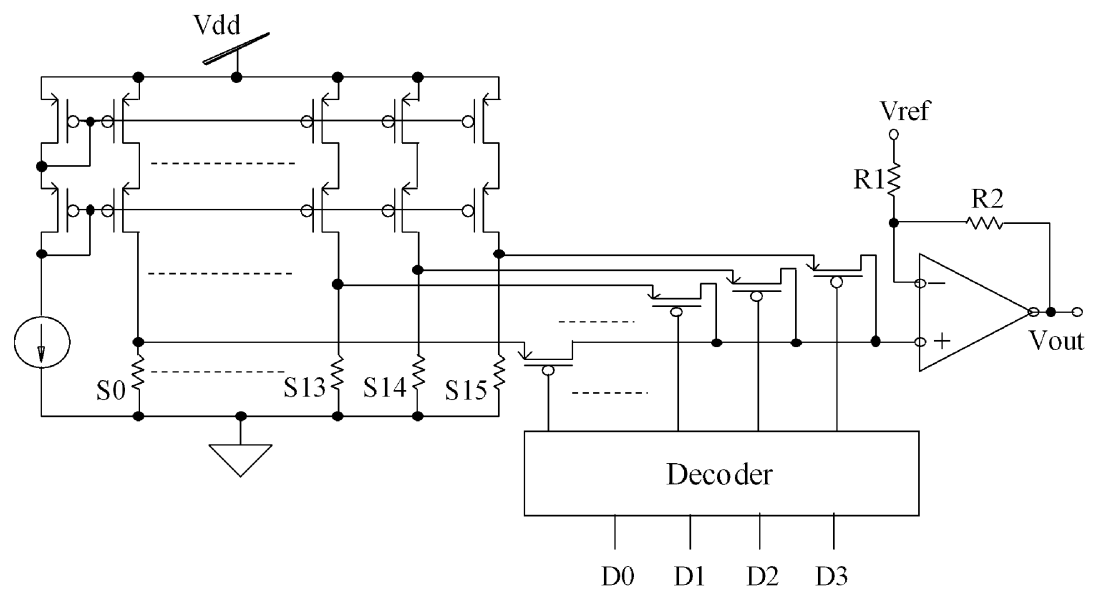

Fig. 3. Schematic of the IC-integrated flexible shear-stress sensor skin.

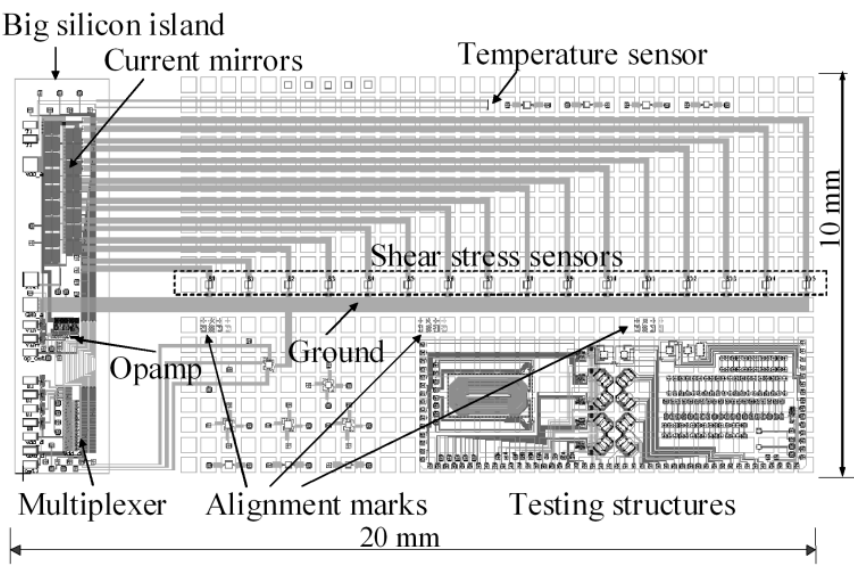

Fig. 4. Layout of the IC-integrated shear-stress sensor skin.

which is very desirable in some specific applications. A temperature sensor is also included for temperature compensation. There are also some testing structures placed on the skin. For example, a coil structure, which can be a micro-flap, is put in the lower-right corner.

\section{FABRICATION}

The circuits are fabricated by Mitel Semiconductor (now Zarlink semiconductor), which is a Canadian CMOS foundry [10], with a $2 \mu \mathrm{m}$ p-well double poly/double metal CMOS process. The gate poly of Mitel process is $320 \mathrm{~nm}$ thick with a temperature coefficient of resistance (TCR) of $0.1 \% /{ }^{\circ} \mathrm{C}$ and a sheet resistance of $20 \Omega$ per square. Fig. 5 shows a six-inch wafer received from Mitel. The dimension of the die is $20 \times 10 \mathrm{~mm}^{2}$. The $y$ step size of dies is chosen as $15 \mathrm{~mm}$ to leave enough silicon as mechanical support when etching through wafer from the backside. The stepper at the Caltech Micromachining Lab uses two alignment marks which are 76.2 $\mathrm{mm}$ apart. As shown in Fig. 4, there are three alignment marks spaced $5.44286 \mathrm{~mm}$ apart on every die. The step size of dies in $x$ direction is chosen as $21.77142 \mathrm{~mm}$ so that there are two alignment marks which are exactly $76.2 \mathrm{~mm}$ apart.

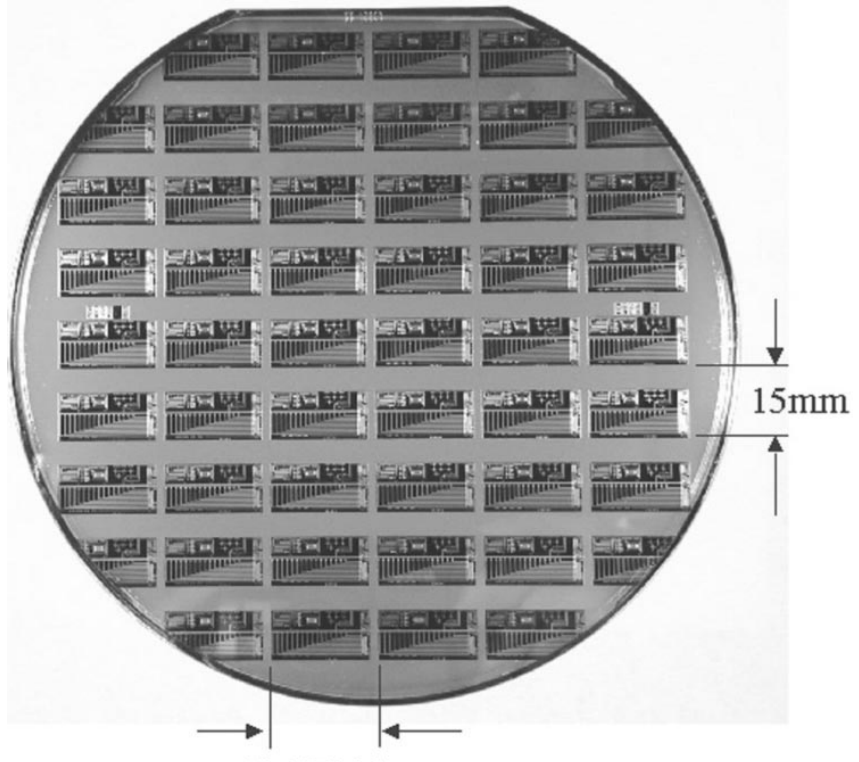

$21.77142 \mathrm{~mm}$

Fig. 5. Photograph of the six-inch wafer received from Mitel.

The post-CMOS process is conducted at the Caltech Micromachining Lab. As Fig. 6(a) shows, after fabricating the circuits at Mitel, the polysilicon sensing elements are covered by dielectric and metal layers. The post-CMOS process begins with patterning the metal and dielectric layers at the sensor area. Then, $10 \mu \mathrm{m}$ parylene $\mathrm{C}$ is deposited and patterned to expose the sensing elements (polysilicon resistors) on the front side of the wafer. Next, $1.5 \mu \mathrm{m}$ parylene $\mathrm{N}$ is also deposited on the front side. The releasing holes for shear-stress sensor are then formed by patterning parylene $\mathrm{N}$ as shown in Fig. 6(d). These holes are further etched into the silicon substrate to enhance the $\mathrm{BrF}_{3}$ releasing process. It is worth noting that both parylene $\mathrm{C}$ and $\mathrm{N}$ are used in this project. Parylene $\mathrm{C}$ serves as the mechanical support to encapsulate the silicon islands and parylene $\mathrm{N}$ serves as the diaphragm material to support the sensing element after releasing. There are two major differences between parylene $\mathrm{C}$ and $\mathrm{N}$ that lead to their different roles. The first difference lies in the melting temperature. Parylene $\mathrm{N}$ has a much higher melting temperature $\left(420^{\circ} \mathrm{C}\right)$ than that of parylene $\mathrm{C}\left(290^{\circ} \mathrm{C}\right)$. Since the 


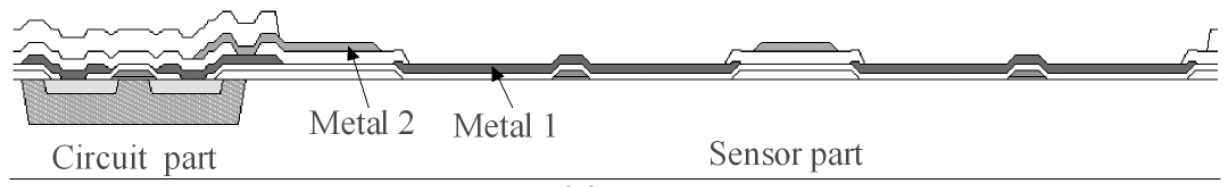

(a)

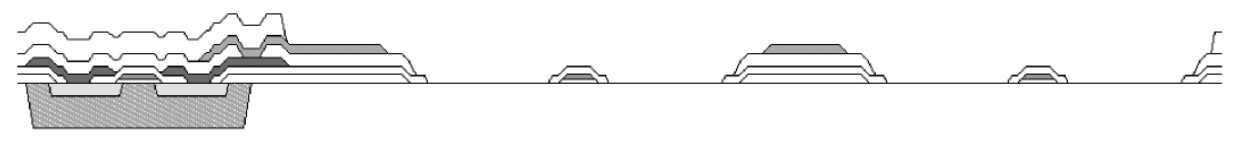

(b)

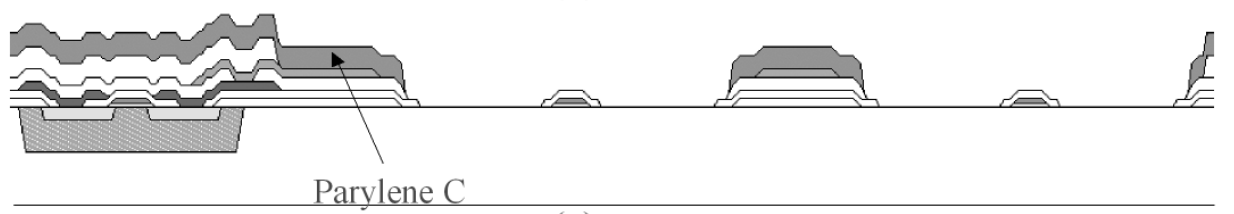

(c)

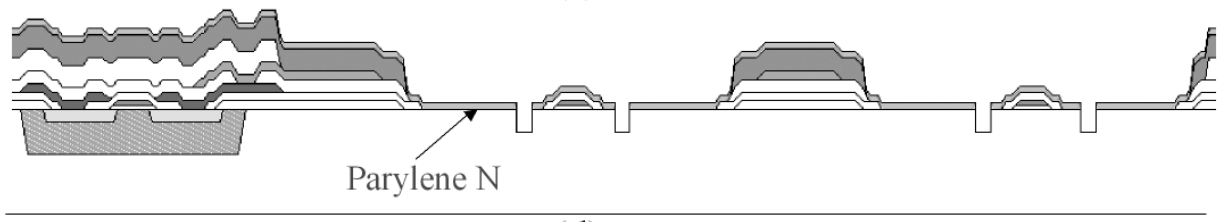

(d)

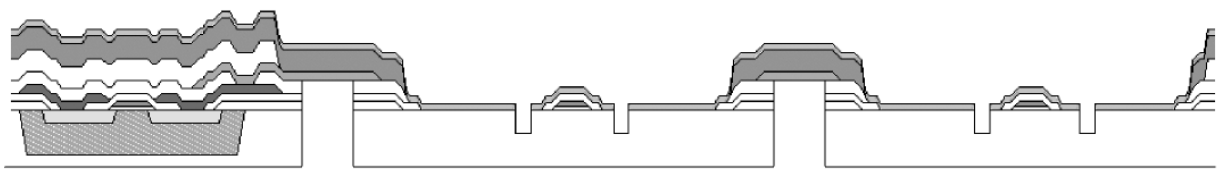

(e)

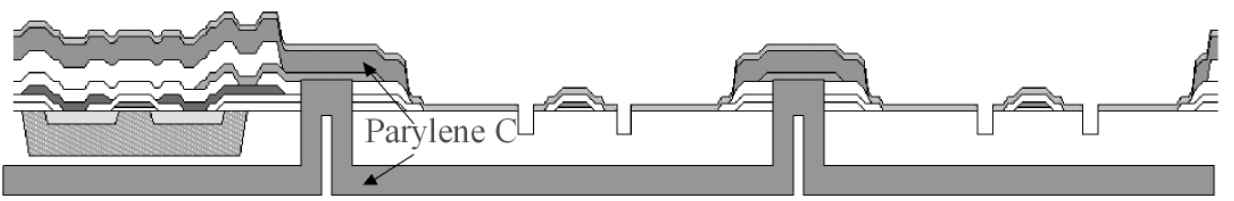

(f)

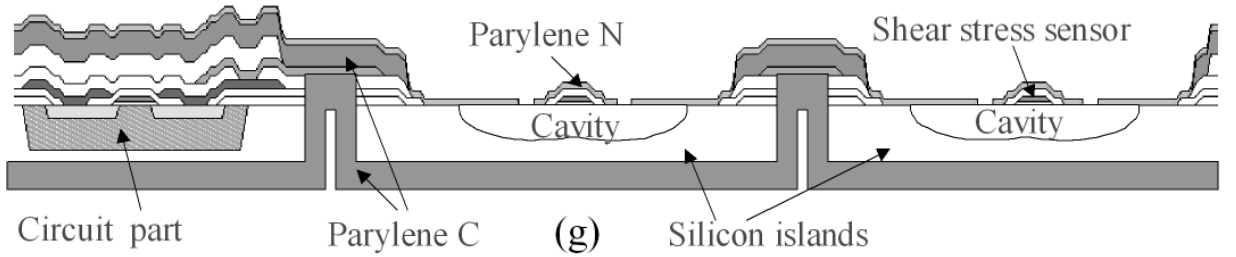

Fig. 6. Simplified post-CMOS fabrication process: (a) Cross section of wafer received from Mitel; (b) Pattern metal and dielectric layers; (c) Deposit and pattern $10 \mu \mathrm{m}$ parylene $\mathrm{C}$ on front side; (d) Deposit and pattern $1.5 \mu \mathrm{m}$ parylene N; (e) Thin down the wafer from back side, form the silicon islands by DRIE, and remove the dielectric layer; (f) Deposit another $10 \mu \mathrm{m}$ parylene $\mathrm{C}$ on backside; (g) Release the shear-stress sensor by $\mathrm{BrF}_{3}$.

sensing element (polysilicon resistor) will be heated up during operation, parylene $\mathrm{N}$ is chosen as the membrane material to ensure the high temperature compatibility. The second difference lies in the deposition rate. The typical deposition rate of parylene $\mathrm{C}$ is $4 \sim 5 \mu \mathrm{m} /$ hour, whereas the deposition rate of parylene $\mathrm{N}$ is about 6 times smaller. To provide enough mechanical support, we need approximately $10 \mu \mathrm{m}$ parylene on both front and back sides. Parylene $\mathrm{C}$ is chosen for its fast deposition rate.

The process at the backside begins with the thinning down of the wafer by DRIE. Next, the silicon islands are formed by selectively etching away silicon by using DRIE again. After etching silicon down to the dielectric layer on the front side, pad etchant is used to remove the dielectric layer. This step is crucial because the dielectric layer, if not removed, will break the aluminum wires on top when the skin undergoes bending. Then, $10 \mu \mathrm{m}$ parylene $\mathrm{C}$ is deposited on the wafer backside to sandwich the silicon islands. The final step is to release the polysilicon resistors by $\mathrm{BrF}_{3}$ gas phase etching. The purpose of this step is to achieve good thermal isolation for the sensing element.

A successfully released shear-stress sensor is shown in Fig. 7(a). There are two columns of releasing holes on each side of the polysilicon resistor. The particulates surrounding 


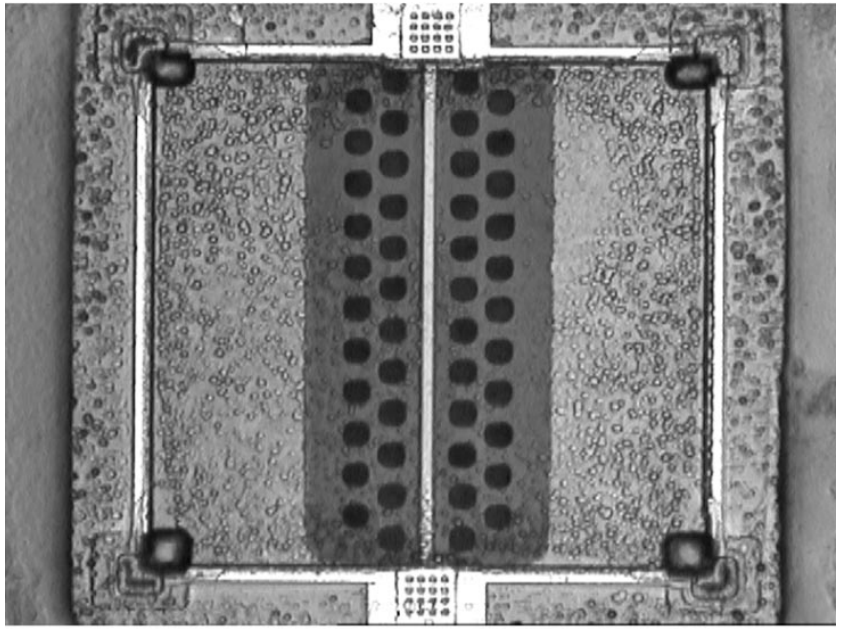

(a)

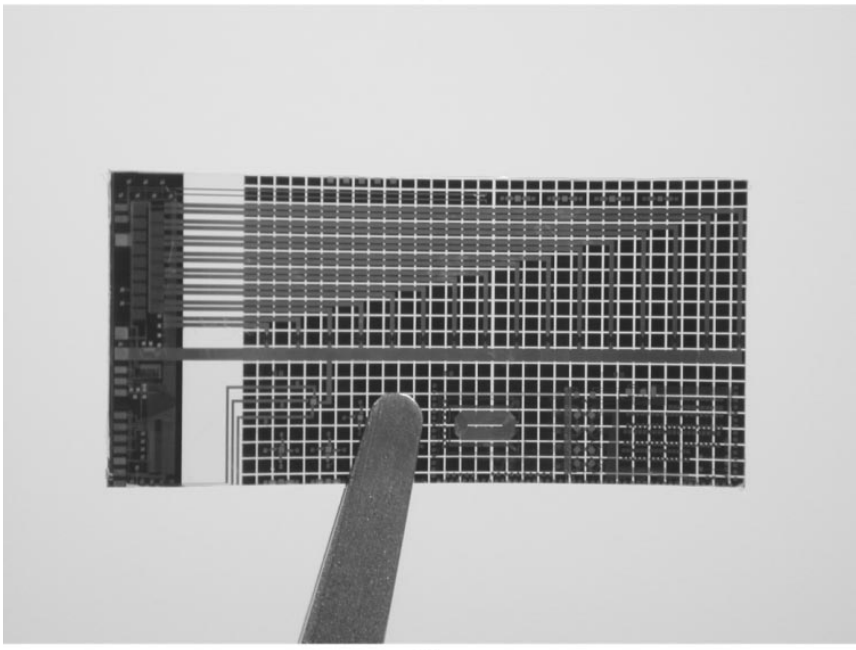

(b)

Fig. 7. (a) Micrograph of a shear-stress sensor released by $\mathrm{BrF}_{3}$; (b) $\mathrm{A}$ fabricated IC-integrated skin $\left(1 \times 2 \mathrm{~cm}^{2}\right)$ held in tweezers.

the sensor are generated during the deposition of $1.5 \mu \mathrm{m}$ parylene $\mathrm{N}$ and are possibly due to a contaminated surface. An IC-integrated sensor skin cut from the wafer is shown in Fig. 7(b). The silicon islands are clearly seen by shining light from the backside. The metal leads going across the islands can also be clearly observed.

\section{PACKAGING}

The sensor skin is very flexible and can be readily mounted on nonplanar surfaces. Fig. 8 shows a single skin packaged on a semicylindrical surface of an aluminum block that is the leading edge of an airfoil. The 16 shear-stress sensors on the skin exactly cover the semicylinder with a diameter of 9.55 mm. Wire-bonding and conventional soldering are used to connect the sensor skin to the outside instrument. This configuration is adequate for our wind tunnel test. However, for real aircraft wing applications, the edge of the skin that contains the metal pads should be folded underneath the wing surface as we demonstrated in [1].

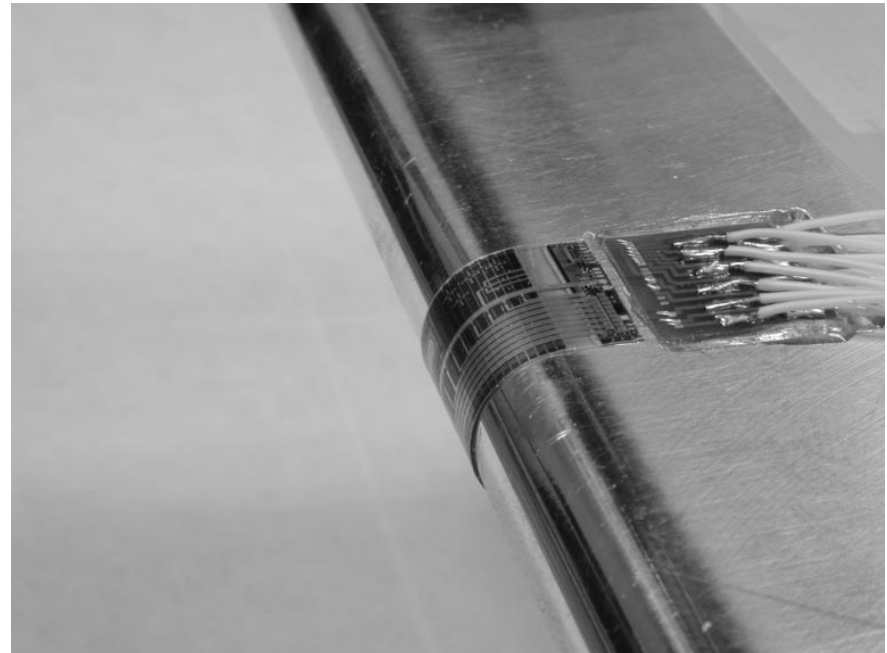

Fig. 8. A sensor skin mounted on a semicylindrical aluminum block.

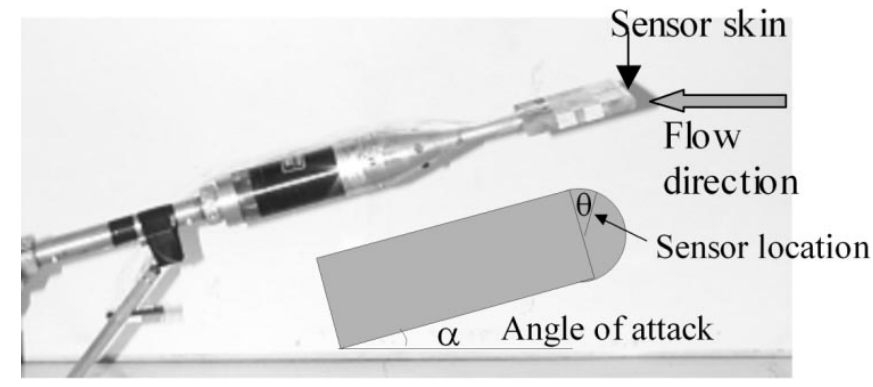

Fig. 9. Testing setup in wind tunnel.

\section{WIND TUNNEL TEST}

The wind tunnel test is carried out in the UCLA wind tunnel lab. The testing setup is illustrated in Fig. 9. The integrated sensor skin is mounted on the top of the semicylindrical aluminum block. The definitions of sensor location $\theta$ and angle of attack $\alpha$ (AOA) can also be found in Fig. 9. The 16 shearstress sensors are biased by current mirrors integrated on-chip. The static voltages (at zero flow) of the sensors are between $2.08 \mathrm{~V}$ and $2.65 \mathrm{~V}$, and the corresponding resistance over-heat ratios are between $12 \%$ and $18 \%$. Since the TCR of polysilicon is $0.1 \% /{ }^{\circ} \mathrm{C}$, the average operating temperatures of sensors are between $140^{\circ} \mathrm{C}$ and $200^{\circ} \mathrm{C}$, which are below the melting temperature of parylene $\mathrm{N}\left(420^{\circ} \mathrm{C}\right)$. In order to compensate this nonuniformity caused by process variation, the measured voltage change $\Delta V$ of every sensor is normalized to its output voltage $V$ [11]. The voltage changes of 16 shear-stress sensors are recorded at different angles of attack and different flow speeds using the on-chip biasing and multiplexing circuitry. The normalized data $(\Delta V / V)$ are summarized in Fig. 10. Sensor 0 is at the top surface $\left(0^{\circ}\right)$ and sensor 15 is at the bottom surface of the aluminum block $\left(180^{\circ}\right)$. The angle between adjacent sensors is $12^{\circ}$ and the 16 sensors exactly span $180^{\circ}$.

The steady-flow separation, which is characterized theoretically by zero shear stress, can be observed. The stagnation point, where the shear stress has a minimum value in the middle, can also be identified. In the case of $0^{\circ} \mathrm{AOA}$, the stagnation point 


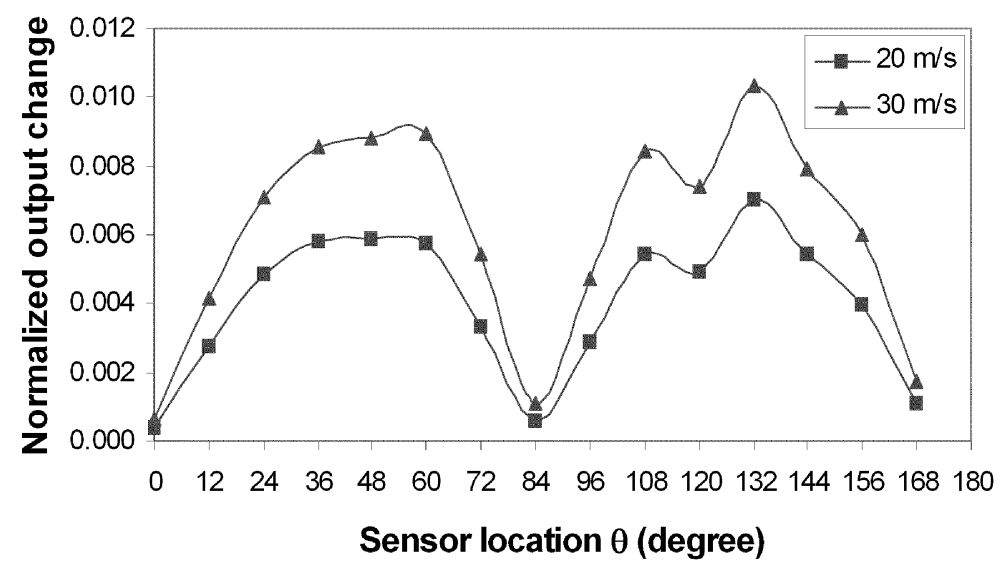

(a) $0^{\circ}$ angle of attack

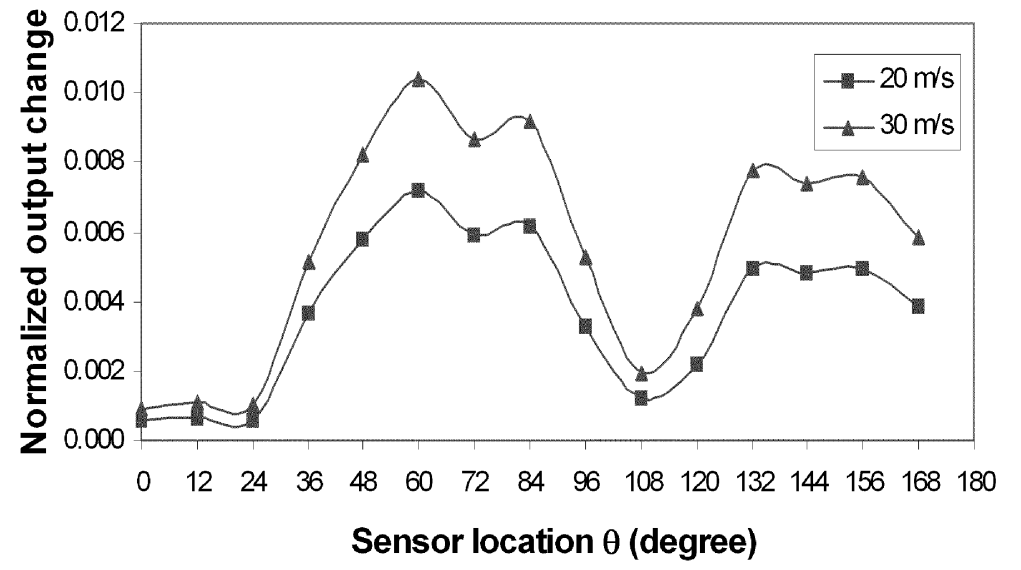

(b) $15^{\circ}$ angle of attack

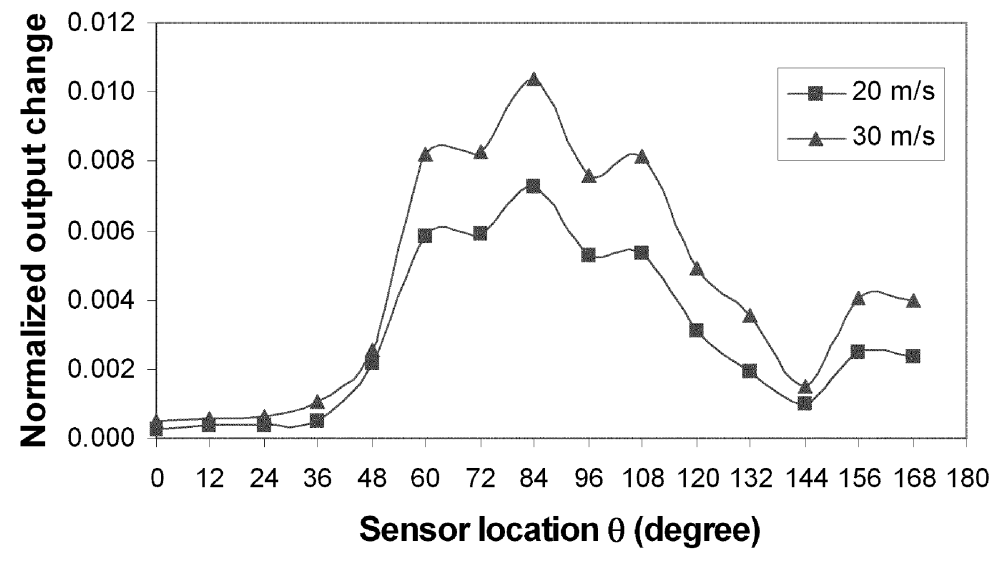

(c) $30^{\circ}$ angle of attack

Fig. 10. Shear-stress distributions at different angles of attack measured by the IC-integrated sensor skin.

is at the sensor $7\left(84^{\circ}\right)$, which is exactly as expected. The flow accelerates away from both sides of the stagnation point, indicated by the rise of shear stress, but starts to decelerate at sensor $3\left(36^{\circ}\right)$ and sensor $11\left(136^{\circ}\right)$ due to the flat surface after the leading edge. In the case of $15^{\circ} \mathrm{AOA}$, the onset of separation is at sensor $5\left(60^{\circ}\right)$ where the flow starts to decrease shear stress rapidly. The stagnation point moves to sensor $9\left(108^{\circ}\right)$ due to the change of AOA. For $30^{\circ} \mathrm{AOA}$, the onset of separation also starts at sensor $5\left(60^{\circ}\right)$, whereas the stagnation point is very near the bottom surface at sensor $12\left(144^{\circ}\right)$. The fact that the onsets of flow separation for both $15^{\circ}$ and $30^{\circ}$ AOAs are the same can be explained by the finite spatial resolution of the sensor array. Nevertheless, the difference of the abruptness of the flow separation can be seen by comparing the slope of the curves. It is also worth noting that the full separation for $15^{\circ} \mathrm{AOA}$ occurs at sensor $2\left(24^{\circ}\right)$ while for $30^{\circ} \mathrm{AOA}$ it occurs at sensor $3\left(36^{\circ}\right)$. 


\section{SUMMARY AND DISCUSSION}

An IC-integrated shear-stress sensor skin is successfully fabricated. With bias and signal conditioning circuitry integrated on-chip, the packaging and deployment of the sensor skin are significantly simplified and the system reliability is improved. Both flow separation and stagnation points have been detected by the sensor skin in the wind tunnel test.

The silicon-based flexible skin technology demonstrated in this paper is very unique. The conventional method to fabricate flexible skins is to directly deposit and pattern thin films on plastic or metal substrates, which is best exemplified by the fabrication of flexible printed circuits boards and thin film transistors. For this conventional method, high temperature processes are ruled out due to the temperature limitation imposed by plastic or metal substrates. The material properties are not optimized and it is very difficult to transplant transducers based on silicon and other rigid materials to flexible substrates. Consequently, the devices that can be fabricated are very limited and performance is usually not optimized. In comparison, the silicon-based flexible skin technology is compatible with the mainstream MEMS and IC technologies. MEMS and ICs can be fabricated first on the silicon wafer before the formation of the skin structure. So far the circuits integrated are only fundamental elements such as current mirror, multiplexer, and operational amplifier. However, there is no fundamental physical reason that a CPU can not be integrated with this technique.

Due to its unique features, the IC-integrated flexible skin technology demonstrated in this paper can have many other interesting applications. Sensor skins that contain physiological sensors can be mounted on human body like a Band-AidTM to monitor the body vital signs. For implantable applications, sensors can be built on flexible substrates to conform to the organ shape or to minimize tissue trauma during patient movement. Smart skins incorporating arrays of tactile, temperature, and other sensors can be very helpful to surgical instruments for minimally invasive surgery. In all these applications, signal processing, conditioning, and transmission circuitry can be integrated to greatly enhance the performance.

\section{ACKNOWLEDGMENT}

The authors would like to acknowledge the help from J. Boland, S. Walker Boland, M. Lige, J. Xie, Z. Han, and T. Roper.

\section{REFERENCES}

[1] F. Jiang, Y. Xu, T. Weng, Z. Han, Y.-C. Tai, A. Huang, C.-M. Ho, and S. Newbern, "Flexible shear stress sensor skin for aerodynamics applications," in Proc. IEEE Int. Conference on Micro Electro Mechanical Systems (MEMS), Miyazaki, Japan, 2000.

[2] P. W. Barth, S. L. Bernard, and J. B. Angell, "Flexible circuit and sensor arrays fabricated by monolithic silicon technology," IEEE Trans. Electron Devices, vol. 32, pp. 1202-1205, 1985.
[3] W. Kuehnel and S. Sherman, "A surface micromachined silicon accelerometer with on-chip detection circuitry," Sens. Actuators A, Phys., vol. 45, pp. 7-16, 1994.

[4] J. H. Smith, S. Montague, J. J. Sniegowski, J. R. Murray, and P. J. McWhorter, "Embedded micromechanical devices for the monolithic integration of MEMS with CMOS," in Proc. IEEE International Electron Devices Meeting, Washington, DC, 1995.

[5] T. J. Hanratty and J. A. Campbell, "Measurement of wall shear stress," in Fluid Mechanics Measurements, 2nd ed, R. J. Goldstein, Ed. New York: Taylor \& Francis, 1996, pp. 575-648.

[6] C. Liu, Y.-C. Tai, J. B. Huang, and C. M. Ho, "Surface micromachined thermal shear stress sensor," in Proc. ASME International Mechanical Engineering Congress and Exposition, Chicago, IL, 1994.

[7] X. Q. Wang, Z. Han, F. Jiang, T. Tsao, Q. Lin, Y.-C. Tai, and C. M. Ho, "A fully integrated shear stress sensor," in Proc. International Conference on Solid-State Sensors and Actuators (Transducer), Sendai, Japan, 1999.

[8] X. Q. Wang, X. Yang, K. Walsh, and Y.-C. Tai, "Gas-phase silicon etching with bromine trifluoride," in Proc. International Conference on Solid-State Sensors and Actuators (Transducer), Chicago, IL, 1997.

[9] Parylene Data Sheet, Specialty Coating System, Indianapolis.

[10] http://www.zarlink.com [Online]

[11] Y. Xu, C.-W. Chiu, F. Jiang, Q. Lin, and Y.-C. Tai, "Mass flowmeter using a multi-sensor chip," in Proc. IEEE International Conference on Micro Electro Mechanical Systems (MEMS), Miyazaki, Japan, 2000.

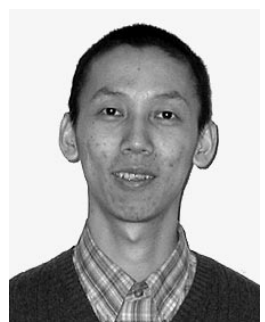

Yong Xu (M'00) received the B.S. degree in electronics engineering from Tsinghua University, Beijing, China, in 1997 and the M.S. and Ph.D. degrees in electrical engineering from the California Institute of Technology, Pasadena, in 1998 and 2002, respectively.

$\mathrm{He}$ is currently an Assistant Professor in the Department of Electrical \& Computer Engineering at Wayne State University, Detroit, MI. His research interests include MEMS smart skin technology, bioMEMS, sensory systems for harsh environments, novel packaging technology, and nanotechnology.

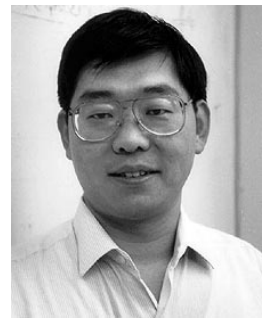

Yu-Chong Tai (M'00) received the B.S. degree from National Taiwan University, Taiwan, and the M.S. and $\mathrm{Ph} . \mathrm{D}$. degrees in electrical engineering from the University of California at Berkeley in 1986 and 1989, respectively.

After Berkeley, he joined the faculty of Electrical Engineering at the California Institute of Technology and built the Caltech Micromachining Laboratory. $\mathrm{He}$ is currently a full Professor of Electrical Engineering at Caltech. His research interests include flexible MEMS, integrated microfluidics, neuroprobes and chips, optical MEMS, and biochemical sensors.

Dr. Tai has received several awards such as the IBM Fellowship, the Best Thesis Award, the Presidential Young Investigator (PYI) Award, and the David and Lucile Packard Fellowship. He co-chaired the 2002 IEEE MEMS Conference, Las Vegas, NV. He is currently a Subject Editor of the JouRnAL OF MicroelectromeChaniCal Systems. He is also a Section Editor of Sensors and Actuators. 


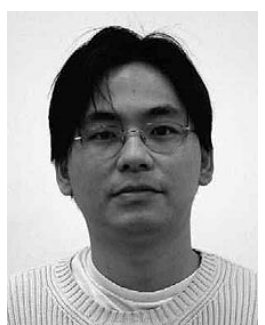

Adam Huang received the B.S. degree in aerospace engineering from the University of California, Los Angeles (UCLA) in 1998. He is currently working toward the Ph.D. degree at UCLA under the guidance of Dr. C.-M. Ho.

His current research topics include MEMS devices for aeronautical engineering and combustion.

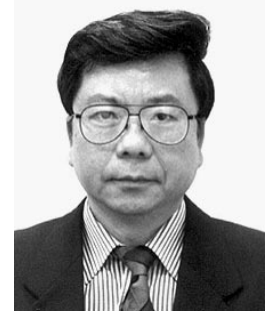

Chih-Ming Ho received B.S. degree from the Mechanical Engineering Department of the National Taiwan University, Taiwan, and the Ph.D. degree from The Johns Hopkins University, Baltimore, MD.

He currently serves as University of California, Los Angeles (UCLA) Associate Vice Chancellor for Research and holds the Ben Rich-Lockheed Martin Chair Professor in School of Engineering. He is the Director of Institute for Cell Mimetic Space Exploration (CMISE). In 1997, he was inducted as a member of the National Academy of Engineering. In 1998, he was elected as an Academician of Academia Sinica. He has published 220 papers and holds seven patents. He is known for his works on micro/nano fluidics, bio/nanotechnology, and turbulence.

Dr. Ho was elected Fellow of the American Physical Society as well as American Institute of Aeronautics and Astronautics for his contributions in a wide spectrum of technical areas. He is a Member of the American Society of Mechanical Engineers (ASME). 